\title{
Medilog - A Social Friendly Android Application for Maintaining Medical Logs And Locating Health Centres
}

\author{
Bhuvaneswari A, Swathi N \\ \{*abh.mca@ psgtech.ac.in, n.swathi97@gmail.com \} \\ Department of Computer Applications, PSG College of Technology Coimbatore 614004 1,2
}

\begin{abstract}
The Medilog project is a mobile application used to store patient medical records. The main objective of the application is patients need not carry their medical records every time while visiting the hospital, patient medical records are stored in this application. In case the doctor wants to see the patient medical record means doctor enters the patient mobile number in this application. OTP is generated to the patient mobile number, then only the doctor can able to view the patient medical records and categorize the medical record based on their wish. This application contains facilitates like show nearby hospitals, it track the patient current location by integrating Google map service, it shows all the nearest hospitals around to the patient. This application provides the feature to the patient to take the pill on time. Once patient uploads their pill-taking details, the application will notify the patient.
\end{abstract}

Keywords- Android application, Patients, Doctors, medical record, nearby location, pill remainder

\section{Introduction}

Medical records are the document that explains all detail about the patient's history, clinical findings, diagnostic test results, pre and postoperative care, patient's progress and medication. Record maintenance is the only way for the doctor to prove that the treatment was carried out properly. Medical records are often the only source of the truth. They are likely to be far more reliable than memory. The management and maintenance of medical records, on the other hand, is a time-consuming job. The remarkable problem is that patients forget to take the proper medicine at the proper time. Medication non- adherence may critically affect the patients.

Nowadays we totally rely on gadgets especially smart phones. Today everyone has a smart phone, in this we get an opportunity to use technology in a better way. To make the job more manageable this application comes down with a motto "Carry medical history in your pocket". Medilog is a personal health record app available for android phones by using this application, it is easy for the patients to store medical records and share the personal medical data with any doctors. The user gets a personalized account for accessing the complete health information. The application assists patients in storing prescriptions and reminds them to take their medications on time. In addition, the app allows patients to be located nearby health hospitals in the event of an emergency or when assistance is needed. 


\section{Existing system}

The various existing applications are Medication remainder, nearby hospital finder, medical records. Patients that use the medication remainder ${ }^{1}$ Application save pills information in the app. It sounds an alarm to alert patients to take their pills on time. The Nearby Hospital Finder ${ }^{2}$ application monitors the patient's current position and recommends all nearby hospitals based on the patient's current location. Medical Records ${ }^{3}$ used to store the medical data, it helps to manage the appointment with the doctors. Challenges

- In Medical records application patients cannot be able to share their records with the doctor.

- $\quad$ Searching the nearby hospital the existing application does not track the patient's current location properly.

- Although exchanging medical records, the application does not retain any authorization

- A single application does not have all of the functionality.

\section{Proposed system}

Medilog application is developed to overcome the problems in the existing application. Patients would be able to upload, classify, and access their medical records in the proposed system depending on their needs. In the event of an emergency, patients would be able to easily find a local hospital. Patients can upload their pill information, and the device will send them an alert to remind them to take their pills on time. Doctors can access a patient's medical records by entering the patient's mobile phone number, which will generate an OTP for the patient. Once patients share, doctors can access their medical history depending on their needs.

Benefits of the application:

- $\quad$ Able to maintain the patient medical record safe and secure.

- It helps the doctor to categorize and view the patient's medical record.

- $\quad$ Get a response from the application quickly

- Show the nearest hospital around to the patient by integrating Google Map service While the doctor has access to the patient report, an OTP is generated and sent to the patient's mobile number. It contributes to the application's protection

\section{System Design}

The Intention of the system design process is to provide sufficiently comprehensive information and data about the system.

This application system flow diagram is shown in Fig 1. Doctors and patients are two parties in the application. Once patients have registered and signed in, they can perform a number of tasks such as uploading medical records, viewing and analyzing those reports, locating nearby locations in the event of an emergency and storing pill information. Entering the patient's mobile number allows the doctor to access the patient's medical records. 


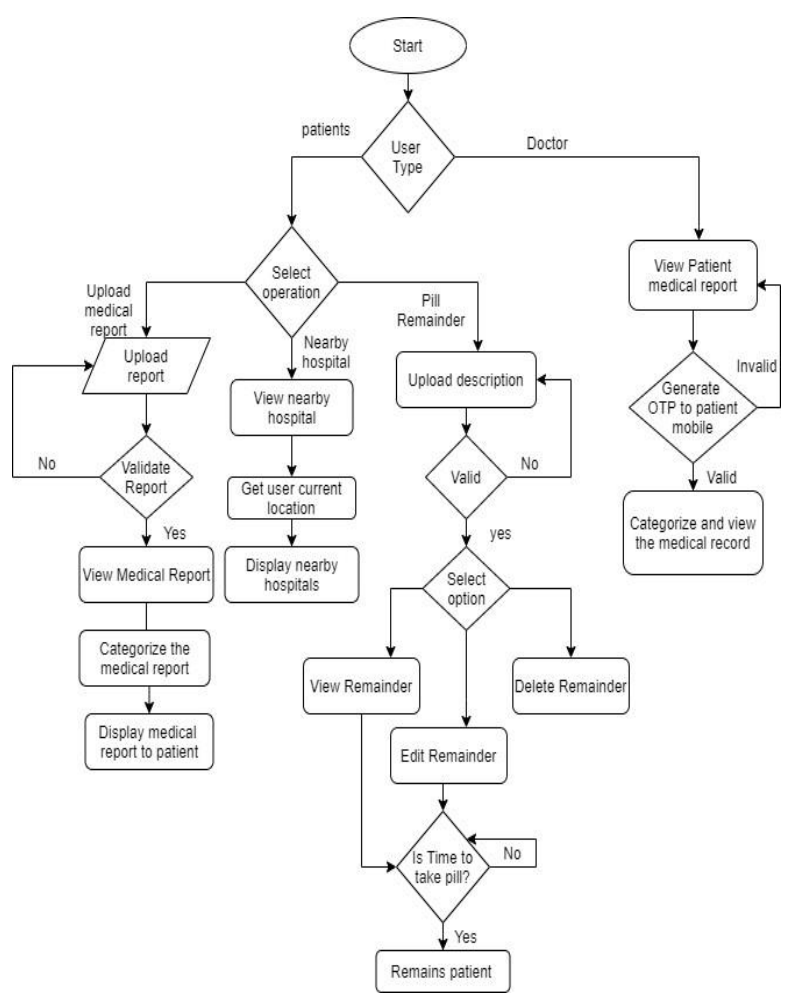

Fig 1: System flow diagram

Fig 2 shows that the patients will submit health records to the patient database even after they have login in to the application. Both the patient and the doctors can view the medical records. Patients must upload their medication information for the pill remainder module, it can be stored in the database, and so that patient can modify or delete the pill details.

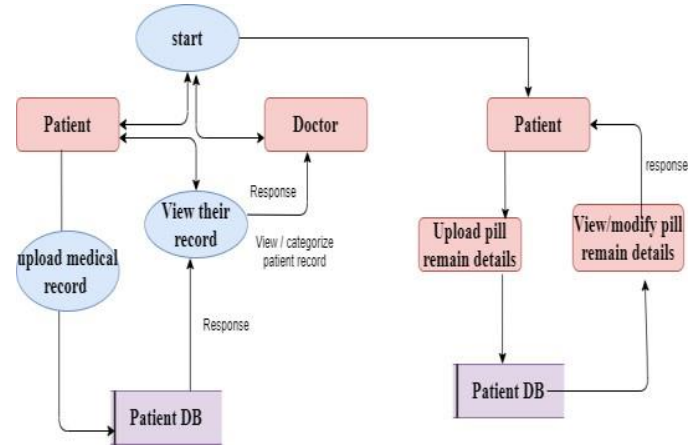

Fig 2: Dataflow Diagram

A.

E-Record 
Patients can upload their medical records by selecting the category and entering the description with their record, to perform some validating methods to check patients entered all fields correctly.

Once the medical record is uploaded patients can able to view, modify, and delete their medical record randomly displaying medical records willet the patient confused and unclear about the report. To avoid such uncertainty, categorization is done.

The categories in the medical reports are to differentiate the records that need to be displayed accordingly. Categories include x-ray, CT-Scan, diagnosis report, doctor prescription, MRI scan, case history, etc. Patients can get the results of viewing their medical records by selecting the category or entering the keyword in the search bar.

\section{B. Nearby Hospital}

Nearby hospital helps the patients to display all the nearby hospital around to the patients, pressing the show nearby hospital button in the application then the application gets the patient's current location by integrating the Google map service.

Then the patient selects the nearby hospital option in the dropdown list, it displays all the nearby hospitals around to the patient.

The tools and technologies used in the development of the Medilog application

\section{Pill Remainder}

The aim of the pill remainder module is to help patients who are busy with remembering to take their daily medications. It is designed for patients who need help to keep track of their medication schedule. The application allows the user to store pill- taking details in this application, based on that application gives alert to the patients. Here patients can able to view the pill details .patients can modify the pill details or delete the pills if they do not need it.

\section{Doctor}

Doctor is another participant in this application the doctor has to enroll in this application. After logging in, type the patient's unique ID (Patient Mobile Number) into the textbox.

Once a mobile number is entered, the doctor has to press the validate button. OTP is generated to the patient's registered mobile number, patient have to tell the OTP with the doctor, then the doctor can able to view the patient medical record.

Randomly displaying medical records will let the doctor confused and unclear of the report. To avoid such uncertainty, categorization is done. It is easier for the doctor to view the patient's medical records or accept user input from search box based on the input, results will be displayed to the doctor.

\section{Tools \& Technologies}

\section{A. Android Studio}

Android is a mobile operating system based on modified version of the Linux Kernel and the opensource software, designed primarily for touch screen mobile devices such as smart phones and tablets. A consortium of developers known as the open handset alliance developed android and it was commercially sponsored by Google Android Studio offers the quickest tools for developing applications for any Android smart phone. It has a gradle-based build framework, an emulator, a code template, and integration with Github.

\section{B. Firebase}

Firebase is a both mobile and web application development platform. It acts as a cloud backend service. It is a real time data base which provides file storage which stores files images to Google cloud storage 
directly from the client. It provides authentication which integrates with the database so the data can be accessed and controlled.

Cloud messaging feature in the firebase is a cross-platform solution for messages and the notifications for Android. Firebase can be used for various purposes but it is mostly used for the real-time database, online processing.

Firebase cloud storage is the most used feature used by developers. Cloud Storage allows developers to quickly and smoothly upload files to a Google Cloud Storage bucket provided and managed by Fire base. Image URL is obtained by uploading an image to a fire base bucket and then that can return back a URL that URL is a permanent URL that can be open anywhere in the firebase.

\section{Implementation}

The development environment, interface configuration, and device implementation are all part of the implementation process

Patients can import their medical records after logging into the application, as shown in Fig 3. The patient should choose an image category and include a report description. All fields are validated by the application, and if validation is successful, the medical records are stored

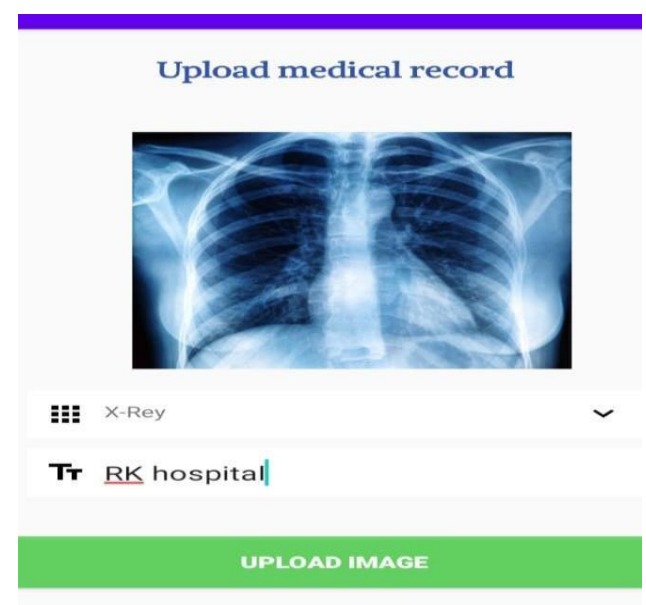

Fig 3: Upload medical records

Doctors can access patient medical records, but they must first enter the patient's mobile number, then OTP has been sent to the patient's registered mobile number as shown in Fig 4, they must share it with the doctor. If the authentication is accurate, then the doctor will be able to access the patient's record.

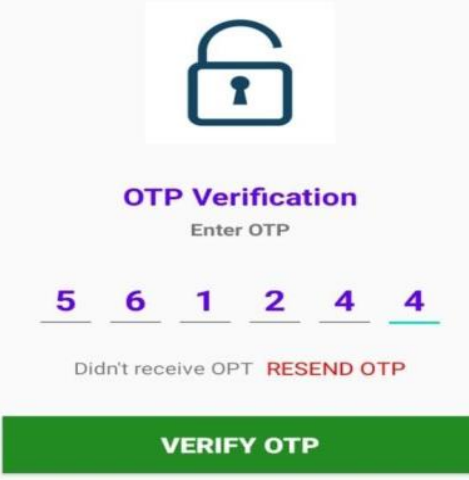




\section{Fig 4 Generate OTP}

Both the patient and the doctor can classify and view the medical record based on their requirements after it has been uploaded as shown in Fig 5, or they can search for the report by typing text into the search bar

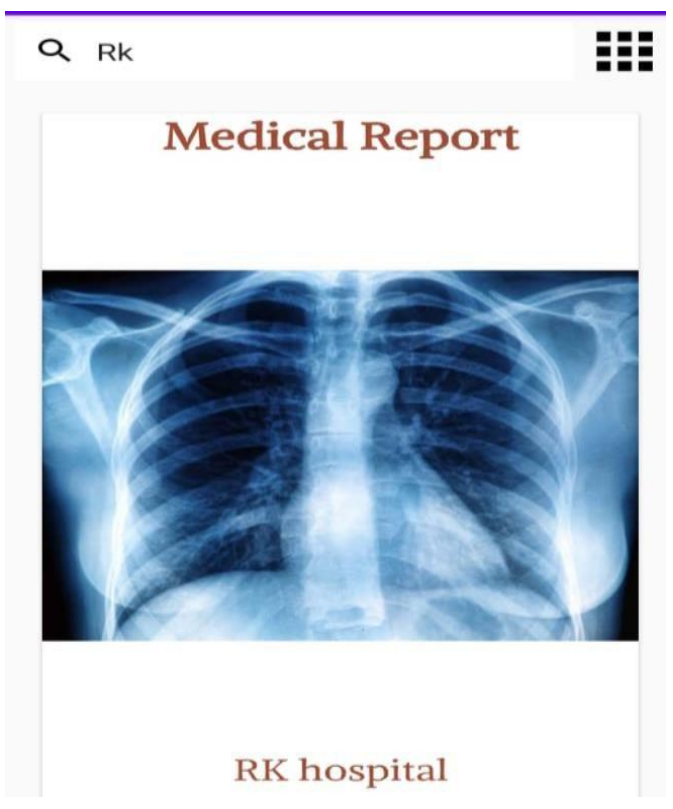

Fig 5: Analyze Medical Report

When patients choose the nearest hospital option during an emergency, the application will show all nearby hospitals in their location as shown in Fig6.

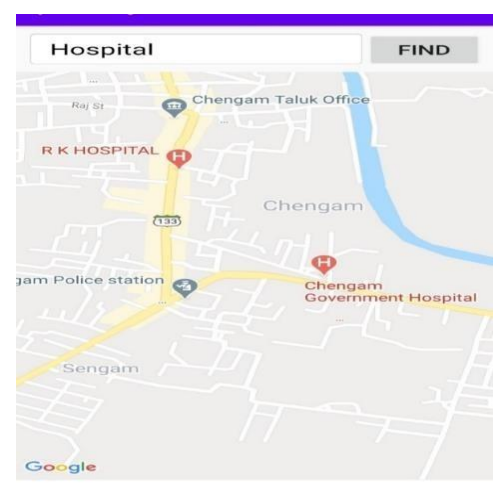

\section{Result}

Fig 6: Nearby Hospital 
The 'medilog' application has a user-friendly interface for patients. Patients who use this application are unable to take their records with them. However they can access them online. Every patient has a collection of documents, which they can categorize according to their needs. This software isn't just for patients; doctors can use it to search their patient's records as well. It assists users in quickly locating the nearest hospital. The application gives reliable remainder good user interface and nice user experience for medication adherence. Hence overall system served well and it truly supports all the features. The doctor's mechanism and functionality are depicted in Figure 7.

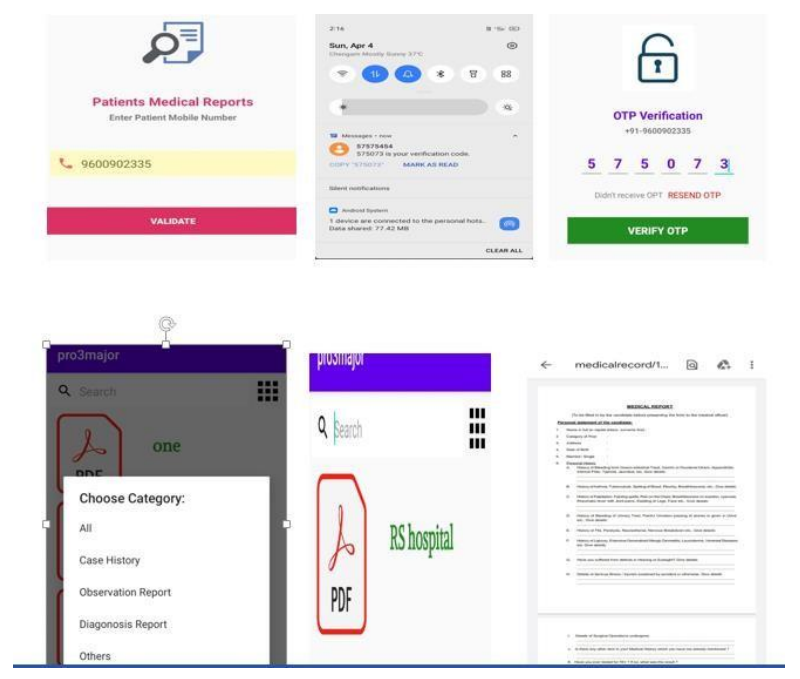

\section{Conclusion And future Work}

Fig 7: Doctor Functionality

Medilog reduces the use of paper records and space occupied to display all the information manually and provides the flexibility in accessing it regardless of time and place. This application both technologically and socially helps patients. This application evolves as a fortune for patients to share their records to doctor at anytime and anywhere. Eventually this application reduces the manual task for both patients and doctors During an emergency it helps to display all the nearby hospital around the patient. Pill remainder reminds the patients about the medicine in-take timings. The proposed system focus on improving the overall performance of health services with advance technologies. Also, the interaction between patients and doctors are through video calling.

\section{REFERENCES}

1. https://play.google.com/store/apps/details?id=com.cliniconline

2. https://play.google.com/store/apps/details?id=com.lions.hospital

3. https://play.google.com/store/apps/deta ils?id=com.devsoldiers.calendar.pills.limit

4. https://developer.android.com/ 